\title{
Cycles and Rationalization
}

\author{
Patrick de Lamirande \\ Financial and Information Management, Cape Breton University, Sydney, Canada \\ Email: Patrick_deLamirande@cbu.ca
}

Received September 27, 2013; revised October 27, 2013; accepted November 4, 2013

Copyright (C) 2013 Patrick de Lamirande. This is an open access article distributed under the Creative Commons Attribution License, which permits unrestricted use, distribution, and reproduction in any medium, provided the original work is properly cited. In accordance of the Creative Commons Attribution License all Copyrights (C) 2013 are reserved for SCIRP and the owner of the intellectual property Patrick de Lamirande. All Copyright (C) 2013 are guarded by law and by SCIRP as a guardian.

\begin{abstract}
This paper studies the composition of the Paretian allocation set in the context of a finite number of agents and a finite number of indivisible goods. Each agent receives at most one good and no monetary compensation is possible (typically called the house allocation problem). I introduce the concept of a cycle which is a sequence of allocations where each allocation is linked to the following allocation in the sequence by the same switch of goods between a subset of agents. I characterize the profiles of agent preferences when the Paretian set has cycles.
\end{abstract}

Keywords: Indivisible Goods; Cycle; Rationalizability; Pareto Allocations

\section{Introduction}

The house allocation problem consists of the assignment of indivisible goods to a set of agents who can receive only one object in the final allocation. Such problems are very common: allocation of rooms between roommates, lectures between professors, offices between colleagues, etc.

This class of problems was introduced by [1]. For this paper, agents own all goods collectively. While authors prove the existence of a competitive equilibrium, [2] shows that this competitive equilibrium is unique when preferences are strict over the set of goods. Reference [3] proves that this unique solution can be implemented by a strategy-proof allocation mechanism. Furthermore, there is a unique strategy-proof, individually rational and Pareto optimal allocation mechanism leading to the unique core allocation [4]. Reference [5] shows the equivalence between the competitive allocation from random endowments and the random serial dictatorship while [6] proves that all mechanisms that are strategy-proof, nonbossy and neutral must be serially dictatorial. Reference [7] models the case where there exists at the same time tenants and new comers on the same market. They introduce the top trading cycles mechanism in this set-up and show that it is Pareto efficient, individually rational and strategy-proof. Reference [8] introduces the possibility of having weak preferences over the set of goods and shows some restrictions on agent preferences for which effi- ciency and coalitional strategy-proofness are compatible ${ }^{1}$.

The purpose of this paper is to look at rationalizability in the context of the house allocation problem. In other words, I am interested in answering the following questions: is it possible to say if, for a given set of allocations, there is a preference profile which supports this set as a Paretian allocation set? An example is students' seats in class. For every lecture, there is an allocation of seats. Considering the set of allocations, it is possible to test the rationality of students' preferences over seats by studying observed allocations.

In existing papers on the house allocation problem, only the [9] mentions explicitly the composition of the Paretian allocation set. They show that for any two allocations in the Paretian set, there exists a sequence of allocations belonging to the Paretian set such that they are pairwise connected, i.e. there are only two agents switching their goods and all others stay with the same good. This means that a set with two allocations that are not pairwise connected cannot be rationalized.

The main difficulty of using direct inference, i.e. testing each possible preference profile if it supports the allocation set as a Paretian allocation set, is linked to number of such preference profiles. As an example, if the number of goods is 5 , then the number of possible allo-

\footnotetext{
${ }^{1}$ This list of papers treating of the house allocation problem is not ex-
} haustive. 
cations is 120 but the number of preference profiles is close to 25 billion $^{2}$. Consequently, it seems reasonable to find a quickest method to study rationalizability.

In this paper, I introduce the concept of a cycle. A cycle is a subset of allocations in which a subset of agents switches their goods according to a specific scheme. The presence of cycles in a given set of allocations which is presumingly a Paretian set gives us information on the potential preference profiles which would support this set as a Paretian allocation set. With the concept of a cycle, I derive some conditions regarding the number of allocations that have to belong to an allocation set in order for it to be a Paretian allocation set.

The paper is organized as follows. In Section 2, I present the house allocation problem and I define the concept of a cycle. Section 4 talks about the properties of the cycle and Section 5 presents the implication of the presence of cycles in the Paretian allocation set. Section 6 concludes.

\section{Definitions and Notations}

Let $N=\{1,2, \cdots, \eta\}$ denote the set of agents with $\eta \geq 2$. The set of goods is $X=\left\{x_{1}, x_{2}, \cdots, x_{\eta}\right\}$ where all goods are different. I define an allocation

$a=\left(a_{1}, a_{2}, \cdots, a_{\eta}\right)$ where $a_{i} \in X$ is the good allocated to agent $i$ with $a_{i} \neq a_{j}$ for $i \neq j$. For any set of agents $\tilde{N} \subseteq N$ and for any set of goods $\tilde{X} \subseteq X$ with $|\tilde{N}|=|\tilde{X}|, A(\tilde{N}, \tilde{X})$ denotes the set of all possible allocations of goods in $\tilde{X}$ to agents in $\tilde{N}$.

Agent $i$ 's preferences are represented by a binary relation $P_{i}$ which is complete, transitive and antisymmetric (strict preference). Given $x_{1}, x_{2} \in X, x_{1} P_{i} x_{2}$ means that agent $i$ strictly prefers $x_{1}$ to $x_{2}$. Also,

$\left.P_{i}\right|_{Y}=\left.P_{j}\right|_{Y}$ means agents $i$ and $j$ have the same preferences over the set of goods $Y$. I define a profile as $P=\left(P_{1}, \cdots, P_{\eta}\right)$ and the domain of all possible profiles is denoted by $\mathbb{P}(N, X)$.

Definition 1: An allocation $a$ is Pareto optimal for a given profile $P$ if $\nexists \hat{a} \in A(N, X)$ such that

$$
\begin{aligned}
& \hat{a}_{i} P_{i} a_{i} \quad \text { for at least one } i \in N \\
& \hat{a}_{j} P_{j} a_{j} \quad \text { or } \hat{a}_{j}=a_{j} \quad \forall j=1,2, \cdots, \eta
\end{aligned}
$$

$P O(P)$ denotes the set of all Paretian allocations when the profile is $P$. Then, $P O(P)$ must be an element of $\mathbb{A}(N, X)$ which is the set of all non-empty subsets of $A(N, X)$. It is important to note here that, for all preference profiles $P$, the set $P O(P)$ is never empty. This means that, for every preference profile $P$, there is at least one allocation which is not Pareto dominated by another allocation.

Definition 2: A set $S$ is rationalizable if there is pre-

${ }^{2}$ The number of possible allocation is given by $n !$ while the number of possible preference profiles is $(n !)^{n}$. ference profile $P$ such that the Paretian allocation set for $P$ is $S$, i.e. $S=P O(P)$.

\section{Cycles}

Since direct inference is at least difficult, it seems natural to look at the structure of the Paretian set to identify some patterns that can be used to find one associated preference profile. Two groups of Paretian sets are trivially easy to rationalize. First, consider a Paretian set with only one allocation. Any preference profile where every agent has the allocated good as his most preferred good rationalizes this Paretian set. The second case is the other extreme case where the Paretian set is composed be all possible allocations. In such case, any preference profile where agents have the same preferences rationalizes this set.

However, intermediate cases are more difficult to infer directly. To solve this problem, I propose the concept of cycle.

Definition 3: Let the set $\tilde{N} \subseteq N$ and $\tilde{X} \subseteq X$ with $|\tilde{N}|=|\tilde{X}|=\tilde{\eta}$. Let $\tilde{n}=\left(\tilde{n}_{1}, \tilde{n}_{2}, \cdots, \tilde{n}_{\tilde{\eta}}\right)$ with $\tilde{n}_{1}, \tilde{n}_{2}, \cdots, \tilde{n}_{\tilde{\eta}} \in \tilde{N}$ and $\tilde{x}=\left(\tilde{x}_{1}, \tilde{x}_{2}, \cdots, \tilde{x}_{e \eta}\right)$ with $\tilde{x}_{1}, \tilde{x}_{2}, \cdots, \tilde{x}_{\tilde{\eta}} \in \tilde{X}$. A set $S \subseteq A(N, X)$ has a cycle $C(\tilde{n}, \tilde{x})$ if $\exists S_{s}=\left\{a^{1}, a^{2}, \cdots, a^{\tilde{\eta}}\right\} \subseteq S$ such that

$$
\begin{gathered}
a_{\tilde{n}_{1}}^{1}=\tilde{x}_{1}, a_{\tilde{n}_{2}}^{1}=\tilde{x}_{2}, \cdots, a_{\tilde{n}_{\tilde{\eta}}}^{1}=\tilde{x}_{\tilde{\eta}} \\
a_{\tilde{n}_{1}}^{2}=\tilde{x}_{2}, a_{\tilde{n}_{2}}^{2}=\tilde{x}_{3}, \cdots, a_{\tilde{n}_{\tilde{\eta}}}^{2}=\tilde{x}_{1} \\
\vdots \\
a_{\tilde{n}_{1}}^{\tilde{\eta}-1}=\tilde{x}_{\tilde{\eta}_{-1}}, a_{\tilde{n}_{2}}^{\tilde{\eta}-1}=\tilde{x}_{\tilde{\eta}}, \cdots, a_{\tilde{n}_{\tilde{\eta}}}^{\tilde{\eta}-1}=\tilde{x}_{\tilde{\eta}-2} \\
a_{\tilde{n}_{1}}^{\tilde{\eta}}=\tilde{x}_{\tilde{\eta}}, a_{\tilde{n}_{2}}^{\tilde{\eta}}=\tilde{x}_{1}, \cdots, a_{\tilde{n}_{\tilde{\eta}}}^{\tilde{\eta}}=\tilde{x}_{\tilde{\eta}-1}
\end{gathered}
$$

For example, if the set $S$ has the cycle $C\left((1,2,3),\left(x_{1}, x_{2}, x_{3}\right)\right)$, this means there are three allocations $a^{1}, a^{2}$ and $a^{3}$ belonging to $S$ such that

$$
\begin{array}{lll}
a_{1}^{1}=x_{1}, & a_{2}^{1}=x_{2}, & a_{3}^{1}=x_{3} \\
a_{1}^{2}=x_{2}, & a_{2}^{2}=x_{3}, & a_{3}^{2}=x_{1} \\
a_{1}^{3}=x_{3}, & a_{2}^{3}=x_{1}, & a_{3}^{3}=x_{2}
\end{array}
$$

It is important to underline that $\tilde{n}$ and $\tilde{x}$ are vectors and not subsets of $N$ and $X$ respectively. The reason is that the order of appearance within the vector is important to the definition of the cycle. To illustrate the importance of this distinction, consider the two following sets:

$$
\begin{aligned}
& S_{1}=\left\{\left(x_{1}, x_{2}, x_{3}\right),\left(x_{2}, x_{3}, x_{1}\right),\left(x_{3}, x_{1}, x_{2}\right)\right\} \\
& S_{2}=\left\{\left(x_{2}, x_{1}, x_{3}\right),\left(x_{1}, x_{3}, x_{2}\right),\left(x_{3}, x_{2}, x_{1}\right)\right\}
\end{aligned}
$$

The set $S_{1}$ has the cycle $C\left((1,2,3),\left(x_{1}, x_{2}, x_{3}\right)\right)$ and $S_{2}$ the cycle $C\left((1,2,3),\left(x_{1}, x_{3}, x_{2}\right)\right)$. But those two cycles are different. For this reason, vectors must be used to define a cycle. 
Also, it should be noted that it is possible to write the same cycle in many ways. Lemma 1 gives the number of ways to write the same cycle ${ }^{3}$.

Lemma 1: Any cycle of $\gamma$ elements can be written in $\operatorname{card}\left(R_{\gamma}\right) \cdot \gamma^{2}$ ways where

$$
R_{\gamma}=\left\{\alpha \in\{1,2, \cdots, \gamma-1\} \mid \nexists \beta<\gamma \text { with } \bmod _{\gamma}(\alpha \beta)=0\right\}
$$

where mod is the modulo operator and $\operatorname{card}\left(R_{\gamma}\right)$ returns the number of elements in $R_{\gamma}$ (cardinality of $\left.R_{\gamma}\right)^{4}$.

The following example illustrates the lemma.

Example 1: Suppose I have the set

$S=\left\{\left(x_{1}, x_{2}, x_{3}\right),\left(x_{2}, x_{3}, x_{1}\right),\left(x_{3}, x_{1}, x_{2}\right)\right\}$. Then, by Lemma 1 , there are $\left(3-\operatorname{card}\left(R_{3}\right)\right) 3^{2}=18$ ways to write the cycle:

$$
\begin{array}{ll}
C\left((1,2,3),\left(x_{1}, x_{2}, x_{3}\right)\right) & C\left((1,2,3),\left(x_{2}, x_{3}, x_{1}\right)\right) \\
C\left((1,2,3),\left(x_{3}, x_{1}, x_{2}\right)\right) & C\left((2,3,1),\left(x_{1}, x_{2}, x_{3}\right)\right) \\
C\left((2,3,1),\left(x_{2}, x_{3}, x_{1}\right)\right) & C\left((2,3,1),\left(x_{3}, x_{1}, x_{2}\right)\right) \\
C\left((3,1,2),\left(x_{1}, x_{2}, x_{3}\right)\right) & C\left((3,1,2),\left(x_{2}, x_{3}, x_{1}\right)\right) \\
C\left((3,1,2),\left(x_{3}, x_{1}, x_{2}\right)\right) & C\left((3,2,1),\left(x_{3}, x_{2}, x_{1}\right)\right) \\
C\left((3,2,1),\left(x_{2}, x_{1}, x_{3}\right)\right) & C\left((3,2,1),\left(x_{1}, x_{3}, x_{2}\right)\right) \\
C\left((2,1,3),\left(x_{3}, x_{2}, x_{1}\right)\right) & C\left((2,1,3),\left(x_{2}, x_{1}, x_{3}\right)\right) \\
C\left((2,1,3),\left(x_{1}, x_{3}, x_{2}\right)\right) & C\left((1,3,2),\left(x_{3}, x_{2}, x_{1}\right)\right) \\
C\left((1,3,2),\left(x_{2}, x_{1}, x_{3}\right)\right) & C\left((1,3,2),\left(x_{1}, x_{3}, x_{2}\right)\right)
\end{array}
$$

It must be noted that $\operatorname{card}\left(R_{\gamma}\right)$ is always higher or equal to 2 when $\gamma$ is higher or equal to 3 . The number 1 and $\gamma-1$ always belong to $R_{\gamma}$.

Since the number of ways to write the same cycle can be large $e^{5}$ I propose using the lexicographic ordering to have a unique notation for a given cycle.

Definition 4: For two vectors $v$ and $w$ of $l$ components, $v$ is lexicographically dominated by $w$ if

$$
\begin{aligned}
w_{1} & >v_{1} \text { or } \\
w_{1} & =v_{1}, w_{2}>v_{2} \text { or } \\
& \vdots \\
w_{1} & =v_{1}, w_{2}=v_{2}, \cdots, w_{l}>v_{l}
\end{aligned}
$$

The first step is to choose from all possibilities of writing a given cycle the ways for which the vector $\tilde{n}$ is lexicographically dominated by (or equal to) the others. Secondly, from those variants, I choose the one for which the component subscripts of $\tilde{x}$ are lexicographically dominated by the other vector $\tilde{x}$.

\footnotetext{
${ }^{3}$ All proofs are in Appendix.

${ }^{4}$ For $\alpha, \beta \in \mathbb{N}, \bmod _{\alpha} \beta$ is the remainder of the division of $\beta$ by $\alpha$.

${ }^{5}$ For example, if $m=5$, then we can write the same cycle in 200 different ways.
}

Let's apply this process to the cycle in Example 1. The first step tells us to select the vector $\tilde{n}$ which is lexicographically dominated by the others. This vector is $(1,2,3)$. Then, from the different ways to write the cycle with $\tilde{n}=(1,2,3)$, which are $C\left((1,2,3),\left(x_{1}, x_{2}, x_{3}\right)\right)$, $C\left((1,2,3),\left(x_{2}, x_{3}, x_{1}\right)\right)$ and $C\left((1,2,3),\left(x_{3}, x_{1}, x_{2}\right)\right)$, we select the one which has the vector $\tilde{x}$ whose component subscripts are lexicographically dominated by the component subscripts of the other $\tilde{x}$ 's. I find that the unique solution is $C\left((1,2,3),\left(x_{1}, x_{2}, x_{3}\right)\right)$.

I most emphasize that the definition of a cycle is independent of what other agents get. For example, consider the two following sets:

$$
\begin{aligned}
& S_{1}= \\
& \left\{\left(x_{1}, x_{2}, x_{3}, x_{4}, x_{5}\right),\left(x_{1}, x_{2}, x_{4}, x_{5}, x_{3}\right),\left(x_{1}, x_{2}, x_{5}, x_{3}, x_{4}\right)\right\} \\
& S_{2}= \\
& \left\{\left(x_{2}, x_{1}, x_{3}, x_{4}, x_{5}\right),\left(x_{2}, x_{1}, x_{4}, x_{5}, x_{3}\right),\left(x_{1}, x_{2}, x_{5}, x_{3}, x_{4}\right)\right\}
\end{aligned}
$$

These sets have the same cycle $C\left((3,4,5),\left(x_{3}, x_{4}, x_{5}\right)\right)$ even if they do not have the same allocations.

An interesting question is how many different cycles could the set $S$ have for set of agents $\tilde{N}$ and a given subset of goods $\tilde{X}$ ? There are $\tilde{\eta}$ ! different vectors $\tilde{n}$ and $\tilde{\eta}$ ! different possible vectors $\tilde{x}$. There are $(\tilde{\eta} !)^{2}$ possibilities. But, I have already shown that there are $\left(\operatorname{card}\left(R_{\tilde{\eta}}\right)\right) \tilde{\eta}^{2}$ ways to write the same cycle. So there are $\frac{(\tilde{\eta}-1) !^{2}}{\operatorname{card}\left(R_{\tilde{\eta}}\right)}$ different cycles for a given subset of agents $\tilde{N}$ and a subset of goods $\tilde{X}$.

Also, it is possible that a Paretian set contains more than one cycle. In particular, it could happen that the Paretian set $P O(P)$ has two cycles: $C(\tilde{n}, \tilde{x})$ and $C(\bar{n}, \bar{X})$ with $\bar{N} \subset \tilde{N}$ and $\bar{X} \subset \tilde{X}$. To examine this case, I define the concept of subcycle.

Definition 5: Suppose that the set $S \subseteq A(X, N)$ has a cycle $C(\tilde{n}, \tilde{x})$. The cycle $C(\bar{n}, \bar{X})$ is a subcycle of $C(\tilde{n}, \tilde{x})$ if $\bar{N} \subset \tilde{N}$ and $\bar{X} \subset \tilde{X}$.

Example 2 illustrates the concept of subcycle.

Example 2: Suppose that the set $S$ has a cycle $C\left((1,2,3,4),\left(x_{1}, x_{2}, x_{3}, x_{4}\right)\right)$. Let $S_{s} \subset S$ is the set of allocations $a^{s}$ belonging to $S$ such that

$$
\begin{aligned}
& a_{1}^{s}=x_{1}, \quad a_{2}^{s}=x_{2}, \quad a_{3}^{s}=x_{3}, \quad a_{4}^{s}=x_{4} \quad \text { or } \\
& a_{1}^{s}=x_{2}, \quad a_{2}^{s}=x_{3}, \quad a_{3}^{s}=x_{4}, \quad a_{4}^{s}=x_{1} \quad \text { or } \\
& a_{1}^{s}=x_{3}, \quad a_{2}^{s}=x_{4}, \quad a_{3}^{s}=x_{1}, \quad a_{4}^{s}=x_{2} \quad \text { or } \\
& a_{1}^{s}=x_{4}, \quad a_{2}^{s}=x_{1}, \quad a_{3}^{s}=x_{2}, \quad a_{4}^{s}=x_{3}
\end{aligned}
$$

Then, this cycle contains 4 different subcycles:

$$
\begin{aligned}
& C\left((1,3),\left(x_{1}, x_{3}\right)\right), C\left((1,3),\left(x_{2}, x_{4}\right)\right), \\
& C\left((2,4),\left(x_{1}, x_{3}\right)\right) \text { and } C\left((2,4),\left(x_{2}, x_{4}\right)\right)
\end{aligned}
$$


To know if the cycle $C(\tilde{n}, \tilde{x})$ has subcycles, I study the set $R_{\tilde{\eta}}$. The next lemma tells us the condition necessary for $C(\tilde{n}, \tilde{x})$ to have subcycles.

Lemma 2: If $\operatorname{card}\left(R_{\tilde{\eta}}\right) \neq \tilde{\eta}-1$, then $C(\tilde{n}, \tilde{x})$ has subcycles.

Example 3: Suppose a set $S$ has the cycle $C(\tilde{n}, \tilde{x})$ with $\tilde{n}=(1,2,3,4,5,6)$ and $\tilde{x}=\left(x_{1}, x_{2}, x_{3}, x_{4}, x_{5}, x_{6}\right)$. This means there are 6 allocations $a^{1}, \cdots, a^{6}$ belonging to $S$ such that:

$$
\begin{gathered}
a_{1}^{1}=x_{1}, a_{2}^{1}=x_{2}, a_{3}^{1}=x_{3}, a_{4}^{1}=x_{4}, a_{5}^{1}=x_{5}, a_{6}^{1}=x_{6} \\
a_{1}^{2}=x_{2}, a_{2}^{2}=x_{3}, a_{3}^{2}=x_{4}, a_{4}^{2}=x_{5}, a_{5}^{2}=x_{6}, a_{6}^{2}=x_{1} \\
\vdots \\
a_{1}^{6}=x_{6}, a_{2}^{6}=x_{1}, a_{3}^{6}=x_{2}, a_{4}^{6}=x_{3}, a_{5}^{6}=x_{4}, a_{6}^{6}=x_{5}
\end{gathered}
$$

Set $R_{6}=\{2,3,4,6\}$. Then, cycle $C(\bar{n}, \bar{x})$ with $\bar{n}=(1,4)$ and $\bar{x}=\left(x_{1}, x_{4}\right)$ is a subcyle of $C(\bar{n}, \bar{x})$.

The last definition about cycles is the following:

Definition 6: The set $S \subseteq A(X, N)$ has a complete cycle $C_{c}(\tilde{N}, \tilde{X})$ with $\tilde{N} \subseteq N$ and $\tilde{X} \subseteq X$ where $\operatorname{card}(\tilde{N})=\operatorname{card}(\tilde{X})$ if for all $\tilde{n}=\left(\tilde{n}_{1}, \tilde{n}_{2}, \cdots, \tilde{n}_{\eta}\right)$ with $\tilde{n}_{1}, \tilde{n}_{2}, \cdots, \tilde{n}_{\tilde{\eta}} \in \tilde{N}$ and $\tilde{X}=\left(\tilde{x}_{1}, \tilde{x}_{2}, \cdots, \tilde{x}_{\tilde{\eta}}\right)$ with $\tilde{x}_{1}, \tilde{x}_{2}, \cdots, \tilde{x}_{\tilde{\eta}} \in \tilde{X}, S$ contains the cycle $C(\tilde{n}, \tilde{x})$.

In other words, the set $T$ has a complete cycle $C_{c}(\tilde{N}, \tilde{X})$ if there is at least one allocation that belongs to $A$ for all possible permutations of goods belonging to $\tilde{X}$ between agents belonging to $\tilde{N}$.

\section{Properties of Cycles and Complete Cycles}

The presence of a cycle $C(\tilde{n}, \tilde{x})$ in a Paretian set $P O(P)$ gives information about the preferences of agents. The first insight given by a cycle is about pairs of goods which are neighbors in the vector $\tilde{X}$.

Proposition 1: If $P O(P)$ has a cycle $C(\tilde{n}, \tilde{x})$, then $\forall i, j \in \tilde{N}$

$$
\begin{aligned}
& \left.P_{i}\right|_{x_{1}, x_{\tilde{\eta}}}=\left.P_{j}\right|_{x_{1}, x_{\tilde{\eta}}} \\
& \text { and }\left.P_{i}\right|_{x_{k}, x_{k+1}}=\left.P_{j}\right|_{x_{k}, x_{k+1}}
\end{aligned}
$$

$\forall k=1,2,3, \cdots, \tilde{\eta}-1$.

Consequently, if the Paretian set has a cycle, then all agents belonging to the cycle have same preferences over any pairs of neighbor goods in that cycle. With this proposition, some information on the associated profile $P$ is provided by the presence of a cycle in the Paretian set. However, information on preferences is only over each pair $\left(x_{k}, x_{k+1}\right)$ and the pair $\left(x_{1}, x_{\tilde{\eta}}\right)$. No information about the preferences over all pairs of goods belonging to the set $\tilde{X}$ can be extracted from the cycle. The following example demonstrates the problem.

Example 4: Suppose the cycle $C\left((1,2,3,4),\left(x_{1}, x_{2}, x_{3}, x_{4}\right)\right)$ belongs to the Paretian set $P O(P)$. Then the following profile supports the cycle.

$$
\begin{array}{llll}
P_{1} & P_{2} & P_{3} & P_{4} \\
x_{1} & x_{3} & x_{1} & x_{3} \\
x_{3} & x_{1} & x_{3} & x_{1} \\
x_{2} & x_{2} & x_{2} & x_{2} \\
x_{4} & x_{4} & x_{4} & x_{4}
\end{array}
$$

Then when the good $x_{3}$ is allocated to someone who belongs to $\{1,3\}$, the good $x_{1}$ is allocated to the other agent in that set. The cycle does not contain an allocation where the good $x_{1}$ is allocated to someone in $\{1,3\}$ and the good $x_{3}$ to someone in $\{2,4\}$. This means that agents in $\{1,3\}$ could have different preferences over the set $\left\{x_{1}, x_{3}\right\}$ than agents in $\{2,4\}$. The same is true for the set of goods $\left\{x_{2}, x_{4}\right\}$.

To analyze preferences over a pair of goods which are not neighbors to each other in the vector $\tilde{X}$, I use the concept of subcycle. In Section 2, I showed that a subcycle is a cycle. So, if a cycle has subcycles, Proposition 1 can be used to infer agents' preferences.

Proposition 2: Suppose $P O(P)$ has a cycle $C(\tilde{n}, \tilde{x})$. For $\alpha \notin R_{\tilde{\eta}}$,

$$
\begin{gathered}
\left.P_{\tilde{n}_{i}}\right|_{\left\{\tilde{x}_{y}, \tilde{x}_{y+\alpha}\right\}}=\left.P_{\tilde{n}_{i+\alpha}}\right|_{\left\{\tilde{x}_{y}, \tilde{x}_{y+\alpha}\right\}} \\
\forall i=1,2, \cdots, \tilde{\eta}-\alpha, \quad \forall y=1,2, \cdots, \tilde{\eta}-\alpha
\end{gathered}
$$

Let's apply this proposition to the following example.

Example 5: Suppose $P O(P)$ has a cycle $C(\tilde{n}, \tilde{x})$ with $\tilde{n}=(1,2,3,4,5,6)$ and $\tilde{x}=\left(x_{1}, x_{2}, x_{3}, x_{4}, x_{5}, x_{6}\right)$. Then, this means there are six allocations $a^{1}, a^{2}, a^{3}, a^{4}, a^{5}, a^{6} \in P O(P)$ such that

$$
\begin{aligned}
& a_{1}^{1}=x_{1}, a_{2}^{1}=x_{2}, \cdots, a_{6}^{1}=x_{6} \\
& a_{1}^{2}=x_{2}, a_{2}^{2}=x_{3}, \cdots, a_{6}^{2}=x_{1} \\
& a_{1}^{3}=x_{3}, a_{2}^{3}=x_{4}, \cdots, a_{6}^{3}=x_{2} \\
& a_{1}^{4}=x_{4}, a_{2}^{4}=x_{5}, \cdots, a_{6}^{4}=x_{3} \\
& a_{1}^{5}=x_{5}, a_{2}^{5}=x_{6}, \cdots, a_{6}^{5}=x_{4} \\
& a_{1}^{6}=x_{6}, a_{2}^{6}=x_{1}, \cdots, a_{6}^{6}=x_{5}
\end{aligned}
$$

Consider goods $x_{1}$ and $x_{4}$. Then,

$$
\begin{array}{ll}
a_{1}^{1}=x_{1}, & a_{4}^{1}=x_{4} \\
a_{1}^{4}=x_{4}, & a_{4}^{4}=x_{1}
\end{array}
$$

Then agents 1 and 4 have same preferences over the $\left\{x_{1}, x_{4}\right\}$. If we continue, we find that

1) Agents in $\{1,2,3,4,5,6\}$ have the same preferences over sets $\left\{x_{1}, x_{2}\right\},\left\{x_{2}, x_{3}\right\},\left\{x_{3}, x_{4}\right\},\left\{x_{4}, x_{5}\right\}$, $\left\{x_{5}, x_{6}\right\}$ and $\left\{x_{1}, x_{6}\right\}$.

2) Agents in $\{1,3,5\}$ have the same preferences over sets $\left\{x_{1}, x_{3}\right\},\left\{x_{2}, x_{4}\right\},\left\{x_{3}, x_{5}\right\},\left\{x_{4}, x_{6}\right\},\left\{x_{1}, x_{5}\right\}$ and $\left\{x_{2}, x_{6}\right\}$.

3) Agents in $\{2,4,6\}$ have the same preferences over sets $\left\{x_{1}, x_{3}\right\},\left\{x_{2}, x_{4}\right\},\left\{x_{3}, x_{5}\right\},\left\{x_{4}, x_{6}\right\},\left\{x_{1}, x_{5}\right\}$ 
and $\left\{x_{2}, x_{6}\right\}$.

4) Agents in $\{1,4\}$ have the same preferences over sets $\left\{x_{1}, x_{4}\right\},\left\{x_{2}, x_{5}\right\}$ and $\left\{x_{3}, x_{6}\right\}$.

5) Agents in $\{2,5\}$ have the same preferences over sets $\left\{x_{1}, x_{4}\right\},\left\{x_{2}, x_{5}\right\}$ and $\left\{x_{3}, x_{6}\right\}$.

6) Agents in $\{3,6\}$ have the same preferences over sets $\left\{x_{1}, x_{4}\right\},\left\{x_{2}, x_{5}\right\}$ and $\left\{x_{3}, x_{6}\right\}$.

This result gives additional information about the profile $P$ since it provides information on preferences over pairs of goods which are not neighbors in the cycle. Subcycles can be analyzed on their own since they are themselves distinct cycles, but they could be supported by different preference profiles across agents than the larger cycle. However, by using subcycles, it is only possible to show that agents which are neighbors in a subcycle have the same preferences over all pairs of goods which are neighbor in this subcycle and it is possible that two distinct subsets of agents in the cycle hold different preferences over the same subset of goods.

While Proposition 2 gives us information about preferences over pairs of goods that are neighbors in a subcycle, Proposition 3 deals with the other pairs.

Proposition 3: Suppose that $P O(P)$ has a cycle $C(\tilde{n}, \tilde{x})$. For all pairs of goods $\tilde{x}_{\alpha}, \tilde{x}_{\beta} \in X$ with $\beta>\alpha$ such that $(\beta-\alpha)$ belongs to $R_{\tilde{\eta}}$,

$$
\left.P_{i}\right|_{\left\{\tilde{x}_{\alpha}, \tilde{x}_{\beta}\right\}}=\left.P_{j}\right|_{\left\{\tilde{x}_{\alpha}, \tilde{x}_{\beta}\right\}} \quad \forall i, j \in \tilde{N}
$$

It must be noted that if $\tilde{\eta}$ is a prime number, all pairs of goods are treated by Proposition 3 since $\left|R_{\tilde{\eta}}\right|=\{\tilde{\eta}-1\}$. In this case, all agents in $\tilde{N}$ have the same preferences over the set $\tilde{X}$.

Corollary 1: If $P O(P)$ has a cycle $C(\tilde{n}, \tilde{x})$ and $\tilde{\eta}$ is a prime number, then $\forall i, j \in \tilde{N}$,

$$
\left.P_{i}\right|_{Y}=\left.P_{j}\right|_{Y}
$$

This result is very strong. Only one cycle is enough to conclude that a subset of agents have the same preferences over a subset of goods. Unfortunately, as showed above, this result cannot be extended to any number of individuals in $\tilde{N}$.

Another case can lead to the conclusion that agents in a subset of $N$ have the same preferences over a subset of goods.

Proposition 4: If $P O(P)$ has a complete cycle $C_{c}(\tilde{N}, \tilde{X})$, the agents in $\tilde{N}$ have the same preferences over $\tilde{X}$.

The presence of a complete cycle gives us more information about agent preferences. In fact, a cycle could give the same information if the number of elements in that cycle is a prime number. Unless it has this characteristic, a cycle by itself does not give information on preferences over all goods. But, if a single cycle cannot give the same information than a complete cycle, many cycles can provide it.

Proposition 5: Let the set $\tilde{N}$ be a subset of $N$ and $\tilde{X}$ a subset of $X$ with $\operatorname{card}(\tilde{N})=\operatorname{card} .(\tilde{X})=\tilde{\eta}$. Let $\alpha$ be the lowest prime number such that $\bmod _{\alpha} \tilde{\eta}=0$. If $P O(P)$ has $\left[\left(\frac{\tilde{\eta}}{\alpha}-1\right) *(\tilde{\eta}-2) !+1\right]$ cycles with same $\tilde{n}$ and same $\tilde{X}$, then the agents in $\tilde{N}$ have the same preferences over $\tilde{X}$.

To illustrate the idea of this proof, consider the following example. Suppose $\operatorname{card}(X)=6$ and suppose

$S$ has the following cycles :

- the 6 cycles given by $C\left((1,2,3,4,5,6),\left(x_{1}, \cdot, x_{2}, \cdot, x_{3}, \cdot\right)\right)$

- the 6 cycles given by $C\left((1,2,3,4,5,6),\left(x_{1}, \cdot, x_{2}, \cdot, x_{4}, \cdot\right)\right)$

- the 6 cycles given by $C\left((1,2,3,4,5,6),\left(x_{1}, \cdot, x_{2}, \cdot, x_{5}, \cdot\right)\right)$

- the 6 cycles given by $C\left((1,2,3,4,5,6),\left(x_{1}, \cdot, x_{2}, \cdot, x_{6}, \cdot\right)\right)$

- the 6 cycles given by $C\left((1,2,3,4,5,6),\left(x_{1}, \cdot, x_{3}, \cdot, x_{2}, \cdot\right)\right)$

- the 6 cycles given by $C\left((1,2,3,4,5,6),\left(x_{1}, \cdot, x_{4}, \cdot, x_{2}, \cdot\right)\right)$

- the 6 cycles given by $C\left((1,2,3,4,5,6),\left(x_{1}, \cdot, x_{5}, \cdot, x_{2}, \cdot\right)\right)$

- the 6 cycles given by $C\left((1,2,3,4,5,6),\left(x_{1}, \cdot, x_{6}, \cdot, x_{2}, \cdot\right)\right)$

If $S$ has only these cycles, this means agents 1,3 and 5 could have different preferences over $x_{1}, x_{2}$ than agents 2,4 and 6 . To have all agents with the same preferences, I must add at least one more cycle.

An interesting question concerning the composition of the Paretian set is what happens to the remaining agents. If the Paretian set has a cycle $C(\tilde{n}, \tilde{X})$, it is interesting to know if there is an allocation in $A(N \tilde{N}, X \tilde{X})$ such that agents outside the cycle get the same goods in all allocations which can constitute the cycle. In other words, if I define $\tilde{X}^{c}=X \tilde{X}, \tilde{N}^{c}=N \tilde{N}$, the question is: "Is there a $z \in A\left(\tilde{N}^{c}, \tilde{X}^{c}\right)$ such that the set $S^{c}$ composed by all allocations belonging to $P O(P)$ where agents in $\tilde{N}^{c}$ get $z$ has a cycle $C(\tilde{n}, \tilde{x})$ ?" The answer is: there is no guarantee that the existence of such an element. Take the following example:

Example 6: Suppose the preferences for 6 agents are given by

$$
\begin{array}{llllll}
P_{1} & P_{2} & P_{3} & P_{4} & P_{5} & P_{6} \\
x_{1} & x_{1} & x_{1} & x_{1} & x_{6} & x_{6} \\
x_{3} & x_{3} & x_{3} & x_{3} & x_{2} & x_{4} \\
x_{2} & x_{4} & x_{2} & x_{4} & x_{5} & x_{5} \\
x_{4} & x_{2} & x_{5} & x_{5} & x_{1} & x_{1} \\
x_{5} & x_{5} & x_{4} & x_{2} & x_{3} & x_{3} \\
x_{6} & x_{6} & x_{6} & x_{6} & x_{4} & y_{2}
\end{array}
$$


Then

$$
\begin{aligned}
& \left(x_{1}, x_{2}, x_{3}, x_{4}, x_{5}, x_{6}\right) \in P O(P) \\
& \left(x_{1}, x_{2}, x_{3}, x_{4}, x_{6}, x_{5}\right) \in P O(P) \\
& \left(x_{2}, x_{3}, x_{4}, x_{1}, x_{5}, x_{6}\right) \notin P O(P) \\
& \left(x_{2}, x_{3}, x_{4}, x_{1}, x_{6}, x_{5}\right) \in P O(P) \\
& \left(x_{3}, x_{4}, x_{1}, x_{2}, x_{5}, x_{6}\right) \in P O(P) \\
& \left(x_{3}, x_{4}, x_{1}, x_{2}, x_{6}, x_{5}\right) \notin P O(P) \\
& \left(x_{4}, x_{1}, x_{2}, x_{3}, x_{5}, x_{6}\right) \in P O(P) \\
& \left(x_{4}, x_{1}, x_{2}, x_{3}, x_{6}, x_{5}\right) \in P O(P)
\end{aligned}
$$

I obtain a cycle $C(\tilde{n}, \tilde{x})$ with $\tilde{n}=(1,2,3,4)$ and $\tilde{x}=\left(x_{1}, x_{2}, x_{3}, x_{4}\right)$. But the subset of $P O(P)$ in which allocations give $x_{5}$ to agent 5 and $x_{6}$ to agent 6 does not contain the cycle $C(\tilde{n}, \tilde{x})$. This is also true for the subset of $P O(P)$ in which allocations give $x_{6}$ to agent 5 and $x_{5}$ to agent 6 .

Example 6 shows that the existence of such an elements is not guaranteed. Nevertheless if such element exists and the agents in $\tilde{N}$ have the same preferences over the set $Y$, then the Paretian set contains a complete cycle $C_{c}(\tilde{N}, \tilde{X})$.

Proposition 6: Let the set $\tilde{N}$ be a subset of $N$ and $\tilde{X}$ a subset of $X$ with $\operatorname{card}(\tilde{N})=\operatorname{card}(\tilde{X})$. Suppose that all agents in $\tilde{N}$ have the same preferences over the set $\tilde{X}$. If the subset of $P O(P)$ composed of allocations in which agents belonging to $\tilde{N}^{c}$ get $z \in A\left(\tilde{N}^{c}, \tilde{X}^{c}\right)$ has the cycle $C(\tilde{n}, \tilde{x})$, then $P O(P)$ has a complete cycle $C_{c}(\tilde{N}, \tilde{X})$ in which $\tilde{N}^{c}$ get $z$.

To illustrate this proposition, consider the case where the Paretian set $P O(P)$ contains the allocations

$\left(x_{1}, x_{2}, x_{3}, x_{4}, x_{5}\right),\left(x_{2}, x_{3}, x_{1}, x_{4}, x_{5}\right)$ and

$\left(x_{3}, x_{1}, x_{2}, x_{4}, x_{5}\right)$. Then, $P O(P)$ has the cycle

$C\left((1,2,3),\left(x_{1}, x_{2}, x_{3}\right)\right)$. By Proposition 6, the allocations $\left(x_{1}, x_{2}, x_{3}, x_{4}, x_{5}\right),\left(x_{2}, x_{1}, x_{3}, x_{4}, x_{5}\right)$ and

$\left(x_{3}, x_{2}, x_{1}, x_{4}, x_{5}\right)$ must also belong to $P O(P)$. Then, $P O(P)$ has a complete cycle $C_{c}\left(\{1,2,3\},\left\{x_{1}, x_{2}, x_{3}\right\}\right)$.

\section{Cycles and Paretian Sets}

Finding a preference profile that rationalizes a set $S$ is easy in some cases. When $S=A(N, X)$, then any preference profile such that agents have same preferences rationalizes the set $S$ has a Paretian set. Also, if the set $S$ has only one allocation $a$, this set can be rationalized by any preference profile in which each agent gets his most preferred good. Even in the case where $S$ has two allocations, this set can be rationalized if the two allocations are pairwise connected. However, it is not possible to go further. At the first look, it is impossible to say if a set $S$ can be rationalized even if all allocations are pairwise connected ${ }^{6}$.

Fortunately, it is possible to find some necessary conditions on rationalizable sets. Before presenting some conditions on rationalizable sets, I need the following proposition.

Proposition 7: Suppose $P O(P)$ has a cycle $C(i, y)$. Let $i$ be an agent belonging to $N$ and $x_{l}$ an element of $\tilde{X}$. If all agents belonging to $\tilde{N}\{i\}$ have the same preferences over the set $\tilde{X}\left\{x_{l}\right\}$, then agents belonging to $\tilde{N}$ have same preferences over $\tilde{X}\left\{x_{l}\right\}$.

The next proposition describes the restrictions on the number of allocations $P O(P)$ must contain.

Proposition 8: If $n \geq 3$ and $P O(P) \neq A(N, X)$, then $\operatorname{card}(P O(P)) \leq(n-1) *(n-1) ! \quad \forall P$. If $|P O(P)|=(n-1) *(n-1) !$, then there exist an agent $i$ and a good $x_{l}$ belonging to $X$ such that there is no allocation $a^{h}$ belonging to $P O(P)$ with $a_{i}^{h}=x_{l}$ and the preference profile is given by

$$
\begin{aligned}
& \text { 1) }\left.P_{g}\right|_{Y}=\left.P_{h}\right|_{Y} \quad \forall g, h \in N \quad Y=X \backslash\left\{x_{l}\right\} \\
& \text { 2) }\left.P_{g}\right|_{X}=\left.P_{h}\right|_{X} \quad \forall g, h \in N \backslash\{i\} \\
& \text { 3) }\left.P_{g}\right|_{\left\{x_{l}, x_{k}\right\}} \neq\left. P_{i}\right|_{\left\{x_{l}, x_{k}\right\}} \forall g N \backslash\{i\} \text { for some } x_{k} \in X \backslash\left\{x_{l}\right\}
\end{aligned}
$$

Using Proposition 8 , it is possible to know if a set cannot be rationalized just by looking to the number of allocations belonging to this set. If

$\operatorname{card}(S) \in[(n-1) *(n-1) !+1, n !-1]$, then there exists no preference profile $P$ such that $S=P O(P)$. But, the reverse is not true. This is a necessary condition. Furthermore, it is possible to use cycles to find other intervals such that, if the number of allocations of a set belongs to those intervals, then this set cannot be rationalized. However, it is not possible to find a general form for all of those intervals.

\section{Conclusions}

The rationalizability in the context of house allocation is hard to provide. Except in cases where there are only a few allocations (1,2 or 3) or for extreme cases (the set of all possible allocations or for singleton), it is very difficult to conclude. The use of cycles can help to analyze the rationalizability of an allocation set.

While Proposition 8 studies the number of elements necessary for an allocation set to be rationalizable, Proposition 6 presents a case where the fact that a set contains a cycle implies that it must contain some specific allocations too. Proposition 8 could be extended to include more conditions, but to devise a complete statement of all cases promises to be very long and complicated. From my point of view, the most interesting ave-

\footnotetext{
${ }^{6}$ For example, consider the set

$T=\left(x_{1}, x_{2}, x_{3}, x_{4}\right),\left(x_{2}, x_{1}, x_{3}, x_{4}\right),\left(x_{1}, x_{2}, x_{4}, x_{3}\right)$. All allocations are pairwise connected but this set cannot be rationalized.
} 
nue for the use of cycles is to employ them like I do in Proposition 6. In short, cycles can be useful to study directly the rationalizability of an allocation set, since by using cycles, it is possible to say if a given allocation set is missing some allocations to be rationalizable.

\section{Acknowledgements}

I would like to acknowledge Mathieu Dufour, Walter Bossert, Lars Ehlers, Yves Sprumont and Yves Richelle for their helpful comments. Of course, all remanning errors are mine.

\section{REFERENCES}

[1] L. Shapley and H. Scarf, "On Cores and Indivisibility," Journal of Mathematical Economics, Vol. 1, No. 1, 1974, pp. 23-37.

http://dx.doi.org/10.1016/0304-4068(74)90033-0

[2] A. E. Roth and A. Postlewaite, "Weak versus Strong Domination in a Market with Indivisible Goods," Journal of Mathematical Economics, Vol. 4, 1977, pp. 131-137. http://dx.doi.org/10.1016/0304-4068(77)90004-0

[3] A. Roth, "Incentive Compatibility in a Market with Indivisibilities," Economic Letters, Vol. 9, 1982, pp. 127-132. http://dx.doi.org/10.1016/0165-1765(82)90003-9

[4] J. Ma, "Strategy-Proofness and the Strict Core in a Market with Indivisibilities," International Journal of Game Theory, Vol. 23, 1994, pp. 75-83. http://dx.doi.org/10.1007/BF01242849

[5] A. Abdulkadiroğlu and T. Sönmez, "Random Serial Dictatorship and the Core from Random Endowments in House Allocation Problems," Econometrica, Vol. 66, No. 3, 1998, pp. 689-701. http://dx.doi.org/10.2307/2998580

[6] L.-G. Svensson, "Strategy-Proof Allocation of Indivisible Goods," Social Choice and Welfare, Vol. 16, 1999, pp 557-567. http://dx.doi.org/10.1007/s003550050160

[7] A. Abdulkadiroğlu and T. Sönmez, "House Allocation with Existing Tenants," Journal of Economic Theory, Vol. 88, 1999, pp. 233-260. http://dx.doi.org/10.1006/jeth.1999.2553

[8] L. Ehlers, "Coalitional Strategy-Proofness House Allocation," Journal of Economic Theory, Vol. 105, 2002, pp. 298-317. http://dx.doi.org/10.1006/jeth.2001.2813

[9] A. Ben-Shoham, R. Serrano and O. Volij, "The Evolution of Exchange," Journal of Economic Theory, Vol. 114, 2004, pp. 310-328.

http://dx.doi.org/10.1016/S0022-0531(03)00112-1 


\section{Appendix}

Proof of Lemma 1: Suppose the set $S$ has a cycle $C(\tilde{n}, \tilde{x})$. Then, the set $S$ contains $\tilde{\eta}$ allocations such that:

$$
\begin{gathered}
a_{1}^{1}=x_{1}, a_{2}^{1}=x_{2}, \cdots, a_{\tilde{\eta}}^{1}=x_{\tilde{\eta}} \\
a_{1}^{2}=x_{2}, a_{2}^{2}=x_{3}, \cdots, a_{\tilde{\eta}}^{2}=x_{1} \\
\vdots \\
a_{1}^{\tilde{\eta}}=x_{\tilde{\eta}}, a_{2}^{\tilde{\eta}}=x_{1}, \cdots, a_{\tilde{\eta}}^{\tilde{\eta}}=x_{\tilde{\eta}-1}
\end{gathered}
$$

I can write this cycle by using $x^{\prime}=\left(x_{2}, x_{3}, \cdots, x_{\tilde{\eta}}, x_{1}\right)$. Then, all cycles $C\left(\tilde{n}, x^{\prime}\right)$ with $x^{\prime}$ which has its components switching neighbor to neighbor relative to $\tilde{X}$ give the same cycle. This gives $\tilde{\eta}$ different ways to write the same cycle. I can do the same thing by switching elements of $\tilde{n}$ and I find also $\tilde{\eta}$ ways to write the cycle. Now, consider the number $r$ which is a positive integer strictly lower than $\tilde{\eta}$. Suppose that there exists no positive integer $q$ strictly lower than $\tilde{\eta}$ such that $\bmod _{\tilde{\eta}} r q=0$. Let

$$
\begin{aligned}
& n^{\prime \prime}=\left(1, r+1, \bmod _{\tilde{\eta}}(2 r)+1, \cdots, \bmod _{\tilde{\eta}}((\tilde{\eta}-1) r)+1\right) \\
& x^{\prime \prime}=\left(x_{1}, x_{r+1}, x_{\bmod _{\tilde{\eta}}(2 r)+1}, \cdots, x_{\bmod _{\tilde{\eta}}((\tilde{\eta}-1) r+1)}\right)
\end{aligned}
$$

and consider the cycle $C\left(n^{\prime \prime}, x^{\prime \prime}\right)$. Since $r$ does not belong to $R_{\tilde{\eta}}$, this means all components of $n^{\prime \prime}$ and $x^{\prime \prime}$ are different. So, the cycle $C\left(n^{\prime \prime}, x^{\prime \prime}\right)$ is the same than $C(\tilde{n}, \tilde{x})$. This is true for all $r$ which are positive integers strictly lower than $\tilde{\eta}$ and do not belong to $R_{\tilde{\eta}}$.

Finally, I obtain $\operatorname{card}\left(R_{\tilde{\eta}}\right) \tilde{\eta}^{2}$.

Proof of Lemma 2: Without lost of generality (WLOG), let's take the cycle $C(\tilde{n}, \tilde{x})$ with $\tilde{n}=(1,2, \cdots, \tilde{\eta})$ and $\tilde{x}=\left(x_{1}, x_{2}, \cdots, x_{\tilde{\eta}}\right)$. If $\operatorname{card}\left(R_{\tilde{\eta}}\right) \neq \tilde{\eta}-1$, it means that there is atleast one $r<\tilde{\eta}$ which does not belong to $R_{\tilde{\eta}}$. If $r$ does not belongs to $R_{\tilde{\eta}}$, this means there is a positive integer $q$ strictly lower than $\tilde{\eta}$ such that $\bmod _{\tilde{\eta}} r q=0$.

Now, consider the vector of agents

$n^{\prime}=\left(1, \rho+1, \bmod _{|I|}(2 \rho)+1, \cdots, \bmod _{|I|}((q-1) \rho)+1\right)$ and the vector of goods

$$
x^{\prime}=\left(x_{1}, x_{\rho+1}, x_{\text {mod }|I|_{\mid l}(2 \rho)+1}, \cdots, x_{\text {mod }_{|I|}((q-1) \rho)+1}\right)
$$

Since $r$ is not equal to $\tilde{\eta}$ and $q$ is strictly lower then $\tilde{\eta}$, the set $N^{\prime}$ is not equal to $\tilde{N}$. I obtain the cycle $C\left(n^{\prime}, x^{\prime}\right)$ which is a subcycle of $C(\tilde{n}, \tilde{x})$.

Proof of Proposition 1: WLOG, suppose that $\tilde{n}=(1,2, \cdots, \tilde{\eta})$ and $\tilde{x}=\left(x_{1}, x_{2}, \cdots, x_{\tilde{\eta}}\right)$. Consider $x_{h}, x_{h+1}$ where $h=1,2, \cdots, \tilde{n}-1$. Suppose that agent 1 prefers $x_{h+1}$ over $x_{h}$. Because $P O(P)$ has the cycle $C(\tilde{n}, \tilde{x})$, there is an allocation belonging to $P O(P)$ such that $x_{h+1}$ is allocated to agent 2 and $x_{h}$ to agent 1 . Since this allocation belongs to $P O(P)$, then agent 2 must also prefer $x_{h+1}$ to $x_{h}$. Again, because $P O(P)$ has the cycle $C(\tilde{n}, \tilde{x})$, there is an allocation belonging to $P O(P)$ such that $x_{h+1}$ is allocated to agent 3 and $x_{h}$ to agent 2. Since this allocation belongs to $P O(P)$, then agent 3 must prefer $x_{h+1}$ to $x_{h}$. If I continue for all agents belonging to $I$, I find that all agents belonging to $\tilde{N}$ must have similar preferences for all pairs $x_{h}, x_{h+1}$ with $h=1,2, \cdots, \tilde{\eta}-1$ and for the pair $x_{1}, x_{\tilde{\eta}}$.

Proof of Proposition 2: WLOG, suppose that $\tilde{n}=(1,2, \cdots, \tilde{\eta})$ and $\tilde{x}=\left(x_{1}, x_{2}, \cdots, x_{\tilde{\eta}}\right)$. Let $q(r)$ be the smallest integer such that, for $r<\tilde{\eta}$ and $r$ not belonging to $R_{\tilde{\eta}}, \bmod _{\tilde{\eta}}(q(r) r)=0$. If $r$ does not belong to $R_{\tilde{\eta}}$, then for every $\gamma=1,2, \cdots, r$ and every $\beta=1,2, \cdots, q(r)-1$, the cycle $C\left(n^{s}, x^{s}\right)$ with

$$
\begin{aligned}
& n^{s}=\left(\gamma, \gamma+r, \gamma+\bmod _{|I|}(2 r), \cdots, \gamma+\bmod _{|I|}((q-1) r)\right) \\
& x^{s}=\left(x_{\beta}, x_{\beta+r}, x_{\beta+\bmod _{|I|}(2 r)}, \cdots, x_{\beta+\bmod _{|I|}((q-1) r)}\right)
\end{aligned}
$$

is a subcyle of $C(\tilde{n}, \tilde{x})$. Because $C\left(n^{s}, x^{s}\right)$ is a subcycle of $C(\tilde{n}, \tilde{x}), P O(P)$ must have the cycle $C\left(n^{s}, x^{s}\right)$. I can then apply Proposition 1 .

Proof of Proposition 3: WLOG, suppose that $\tilde{n}=(1,2, \cdots, \tilde{\eta})$ and $\tilde{x}=\left(x_{1}, x_{2}, \cdots, x_{\tilde{x}}\right)$. Now, take $x_{\alpha}$ and $x_{\beta}$ with $\alpha=1,2, \cdots, \tilde{\eta}-1$ and $\beta=\alpha+1, \cdots, \tilde{e} t a$ and let $\delta=\beta-\alpha$. By assumption, $\delta$ belongs to the set $R_{\tilde{\eta}}$.

Suppose that $x_{\alpha} \quad P_{1} x_{\beta}$. Because $P O(P)$ has the cycle $C(\tilde{n}, \tilde{x})$, there is an allocation belonging to $P O(P)$ such that $x_{\alpha}$ is allocated to agent $\delta+1$ and $x_{\beta}$ to agent 1 . Since this allocation belongs to $P O(P)$, then agent $\delta+1$ must prefer $x_{\alpha}$ to $x_{\beta}$. Again, because $P O(P)$ has the cycle $C(\tilde{n}, \tilde{x})$, there is an allocation belonging to $P O(P)$ such that $x_{\alpha}$ is allocated to agent $\bmod _{\tilde{\eta}}(2 \delta)+1$ and $x_{\beta}$ to agent $\delta+1$. Since this allocation belongs to $P O(P)$, then agent $\bmod _{\tilde{\eta}}(2 \delta)+1$ must prefer $x_{\alpha}$ to $x_{\beta}$.

I can continue until I show that

$$
\begin{array}{lll}
x_{\alpha} & P_{\gamma} & x_{\beta}
\end{array}
$$

with

$$
\gamma=1, \delta+1, \bmod _{\tilde{\eta}}(2 \delta)+1, \cdots, \bmod _{\tilde{\eta}}((\tilde{\eta}-1) \delta)+1
$$

Since there is no positive integer $q<\tilde{\eta}$ such that $\bmod _{\tilde{\eta}}(\delta q)=0$, then the set $\left\{1, \delta+1, \bmod _{\tilde{\eta}}(2 \delta)+1, \cdots, \bmod _{\tilde{\eta}}((\tilde{\eta}-1) \delta)+1\right\}$ has $\tilde{\eta}$ elements. So all agents belonging to $\tilde{N}$ have the same preferences over the set $\left\{x_{\alpha}, x_{\beta}\right\}$.

Proof of Proposition 4: Suppose the opposite is true, i.e. there exists $i, j \in \tilde{N}$ and $x^{\prime}, x^{\prime \prime} \in \tilde{X}$ such that

$$
\begin{array}{llll}
x^{\prime} & P_{i} & x^{\prime \prime} \\
x^{\prime \prime} & P_{j} & x^{\prime}
\end{array}
$$

This means the good $x^{\prime}$ will never be allocated to $j$ 
when the good $x^{\prime \prime}$ is allocated to $i$. That contradicts the existence of a complete cycle.

Proof of Proposition 5: Suppose $\tilde{\eta}$ is prime. By Corollary 1 , if $P O(P)$ has a cycle $C(\tilde{n}, \tilde{x})$, then all agents belonging to $\tilde{N}$ have the same preferences over the set $\tilde{X}$.

Now, suppose $\tilde{\eta}$ is not a prime number and let $\alpha$ be the smallest prime number higher than 1 such that $\bmod _{\alpha}(\tilde{\eta})=0$.

Suppose $x_{1}, x_{2} \in \tilde{X}$. Let $N^{1}$ and $N^{2}$ be two nonempty subsets of $\tilde{N}$ such that all agents belonging to $N^{1}$ prefer goods $x_{1}$ to $x_{2}$ and all agents belonging to $N^{2}$ prefer goods $x_{2}$ to $x_{1}$.

Suppose $P O(P)$ has $\tau$ cycles $C\left(\tilde{n}, \tilde{x}^{t}\right)$ for $t=1,2, \cdots, \tau$. By convention, $\tilde{x}_{1}^{t}=x_{1}$ for all $t$. For all cycles $C\left(\tilde{n}, \tilde{x}^{t}\right)$, I define $\beta^{t}$ the positions of $x_{2}$ in the vector $\tilde{v}^{t}$ so that $\tilde{x}_{\beta}^{t}=x_{2}$, and let $\delta^{t}=\beta^{t}-1$.

By Proposition 3, if there is a $t$ such that $\delta^{t}$ does not belong to $R_{\tilde{\eta}}$, then all agents must have the same preferences. Suppose $\delta^{t}$ belongs to $R_{\tilde{\eta}}$ for all $t$. Let the set $\Pi$ be equal to $\{\alpha, 2 \alpha, \cdots, \tilde{\eta}-\alpha\}$ which is a subset of $R_{\tilde{\eta}}$.

The maximum number such that all $\delta^{t}$ belong to $\Pi$ is $\left(\frac{\tilde{\eta}}{\alpha}-1\right)(\tilde{\eta}-2) !$. If $\tau=\left(\frac{\tilde{\eta}}{\alpha}-1\right)(\tilde{\eta}-2)$ !, then there is at least one cycle $C\left(\tilde{n}, \tilde{X}^{t}\right)$ with $\delta^{t}=\alpha$. By Proposition 2, then agents belonging to the same set

$j, j+\alpha, 1+2 \alpha, \cdots, j+(\tilde{\eta}-\alpha) \quad$ for $\quad j=1,2, \cdots, \alpha$ have the same preferences. But, agents in different subsets could have different preferences.

If I add another cycle $C\left(\tilde{n}, \tilde{x}^{\theta}\right)$, then $\delta^{\theta}$ does not belong to $\Pi$. If $\delta^{\theta}$ does not belong to $R_{\tilde{\eta}}$, by Proposition 3, all agents must have the same preferences over $\left\{x_{1}, x_{2}\right\}$. If $\delta^{\theta}$ belongs to $R_{\tilde{\eta}}$, by Proposition 1, for $h=1,2, \cdots, \delta^{\theta}$, agents belonging to

$h, h+\delta^{\theta}, 1+\bmod _{\tilde{\eta}}\left(2 \delta^{\theta}\right), \cdots, h+\left(\tilde{\eta}-\delta^{\theta}\right)$ have the same preferences. Since $\delta^{\theta}$ does not belong to $\Pi$, then $h$ and $h+\delta^{\theta}$ does not belong to the same set

$j, j+\alpha, 1+2 \alpha, \cdots, j+(\tilde{\eta}-\alpha)$ for $j=1,2, \cdots, \alpha$. So the two sets which contain agent $h$ and $h+\delta^{\theta}$ must have the same preferences. I can continue to conclude that all agents must have the same preferences.

Proof of Proposition 6: WLOG, suppose that

$\tilde{X}=\left\{x_{1}, x_{2}, \cdots, x_{\tilde{\eta}}\right\}$ and $\tilde{N}=\{1,2, \cdots, \tilde{\eta}\}$. Let the set

$X^{c}$ be equal to $X \tilde{X}$ and $N^{c}=N \tilde{N}$.

Now suppose that $a \notin P O(P)$ where agents belonging to $\tilde{N}$ get goods belonging to $\tilde{X}$. This means there exists an allocation $b \in A(X, I)$ such that

$$
\begin{aligned}
& b_{i} P_{i} a_{i} \quad \text { for at least one } i \\
& b_{j} P_{j} a_{j} \quad \text { or } b_{j}=a_{j} \quad j=1,2, \cdots,|N|
\end{aligned}
$$

There are three possible cases for the allocation $b$. The first case consists of a reallocation between agents in
$N^{c}$.

But this kind of reallocation cannot Pareto dominate the allocation $a$ because there exists an allocation $\tilde{a}$ belonging to the Paretian set in which agents belonging to $N^{c}$ get $z$. If a reallocation between agents in $N^{c}$ dominates $a$, then the allocation $\tilde{a}$ should not belong to $P O(P)$.

The second case is a reallocation between agents in $N$. Again, it is not possible for this new allocation to dominate the allocation $a$. I assume that all agents in $N$ have the same preferences over goods in $X$. Then no reallocation between agents in $N$ could Pareto dominate the allocation $a$.

Finally, the last possibility is a reallocation between agents in both sets Because the agents in $N$ have the same preferences, $y_{1}$ is preferred to $y_{k}$ by all agents in $N$.

Suppose the agent who gets $y_{1}$ in the new allocation is agent $\alpha$. Because of the cycle, there is an allocation in this cycle such that $\alpha$ gets good $y_{k}$. Then this allocation could not be in the Paretian set because this allocation will be dominated.

This means that the allocation where $\alpha$ gets $y_{k}$ is Pareto dominated and contradicts the existence of a cycle $C(\tilde{n}, \tilde{x})$ in the set $S$.

Proof of Proposition 7: For all pairs of goods belonging to $\tilde{X}\left\{x_{\gamma}\right\}$, I can apply Proposition 2 or Proposition 3 to find that there is at least one agent belonging to $\tilde{N}\{\delta\}$ with the same preferences as $\delta$. Because all agents belonging to $\tilde{N}\{\delta\}$ have the same preferences over $\tilde{X}\left\{x_{\gamma}\right\}$, then all agents belonging to $\tilde{N}$ have the same preferences over $\tilde{X}\left\{x_{\gamma}\right\}$.

Proof of Proposition 8: Let $\Psi=A(N, X) P O(P)$. By assumption, $|\Psi| \leq(n-1)$ ! .

Step 1: Consider the good $x_{1}$. Suppose that agent 1 gets good $x_{1}$ the least often in the allocations belonging to $\Psi$. Then, the number of allocations in $\Psi$ where agent 1 gets $x_{1}$ is less than

$$
\frac{(\eta-1) !}{\eta}
$$

which is strictly lower than $(\eta-2) !$. This means there is at least one cycle $C\left(n^{1}, x^{1}\right)$ with $n^{1}=(2,3, \cdots, \eta)$ and $X^{1}=\left\{x_{2}, x_{3}, \cdots, x_{\eta}\right\}$ since there are exactly $(\eta-2)$ ! of such cycles.

Now take $x_{2}$. Again WLOG, suppose that $x_{2}$ is the good which is the least assigned to agent 2 in the set $\Psi$ when good $x_{1}$ is assigned to agent 1 . The number of allocations in this case is less than

$$
\frac{(\eta-2) !}{(\eta-1)}
$$

which is strictly lower than $(\eta-3)$ !. This means there is at least one cycle $C\left(n^{2}, x^{2}\right)$ with $n^{2}=(3, \cdots, \eta)$ and 
$x^{2}=\left\{x_{3}, \cdots, x_{n^{2}}\right\}$ since there are exactly $(\eta-3) !$ of such cycles.

I can continue until $\eta-t-1$ is a prime number. Let $x_{\alpha}$ belong to $\left\{x_{t}, x_{t+1}, \cdots, x \eta\right\}$ and suppose that agent $\alpha$ gets good $x_{\alpha}$ the most often in the allocations belonging to $P O(P)$ when $x_{1}$ is allocated to agent 1 , $x_{2}$ to agent $2, \cdots, x_{t-1}$ to agent $t-1$. Then, by Corollary 1 , all agents who belong to $\{t, t+1, \cdots, \eta\}\{\alpha\}$ have the same preferences over the set $\left\{x_{t}, x_{t+1}, \cdots, x_{\eta}\right\}\left\{x_{\alpha}\right\}$.

Step 2: Now, consider the general case where agents in $\{s, s+1, \cdots, \eta\} \beta$ have the same preferences over the set $\left\{x_{s}, x_{s+1}, x_{s+2}, \cdots, x_{\eta}\right\}\left\{x_{\beta}\right\}$. But there is at least one cycle $C(\tilde{n}, \tilde{x})$ with $\tilde{n}=(s, s+1, \cdots, \eta)$ and $\tilde{x}=\left\{x_{s}, x_{s+1}, x_{s+2}, \cdots, x_{\eta}\right\}$. By Proposition 7, all agents belonging to $\tilde{N}$ must have the same preferences over the set $\left\{x_{s}, x_{s+1}, x_{s+2}, \cdots, x_{\eta}\right\}\left\{x_{\beta}\right\}$.

Step 3: I can use the same approach with the two remaining $x_{\alpha}$. Doing so, I find that all agents belonging to $\{s, s+1, \cdots, \eta\}$ have the same preferences over the set $\left\{x_{s}, x_{s+1}, x_{s+2}, \cdots, x_{\eta}\right\}$.

I use this approach until I find that all agents belonging to $\{2,3, \cdots, \eta\}$ have the same preferences over the set $\left\{x_{2}, x_{3}, \cdots, x_{\eta}\right\}$.

Step 4: If $|\Psi|$ is strictly lower than $(n-1)$ !, this means there is at least one cycle $C(n, x)$, Then, by Proposition 7, all agents have the same preferences over the set $\left\{x_{2}, x_{3}, \cdots, x_{\eta}\right\}$. Now, if steps 1 to 3 are done once again with $x_{2}$ and $x_{3}$ instead of $x_{1}$, it can be seen that all agents must have the same preferences over the set $\left\{x_{1}, x_{2}, x_{3}, \cdots, x_{\eta}\right\}$.

Step 5: Now suppose that $|\Psi|$ is equal to $(\eta-1)$ !. Suppose that there are two allocations $a^{1}$ and $a^{2}$ belonging to $\Psi$ such that all agents get different goods, there is no $\alpha \in\{1,2, \cdots, \eta\}$ such that $a_{\alpha}^{1}=a_{\alpha}^{2}$.

Let the vector $i$ be the cycle of goods from $a^{1}$ to $a^{2}$. In other words, the good allocated to agent $i_{\alpha}$ in the allocation $a^{1}$ goes to agent $i_{\alpha+1}$. Since there are two allocations composing the same cycle $C(n, x)$ and there are $(\eta-1)$ ! allocations, this means there is at least one cycle and I obtain that all agents must have the same preferences.

The only way to avoid the possibility of having a cycle of $\eta$ elements in the set $P O(P)$ is for all allocations belonging to $\Psi$, there is a good which is never allocated to an agent.

Suppose this good is $x_{\gamma}$ and the agent never getting $x_{\gamma}$ in $P O(P)$ is $\delta$. Since all allocations belong to $P O(P)$, all agents have the same preferences over the set $X\left\{x_{\gamma}\right\}$ and all agents belonging to $I\{\delta\}$ have the same preferences over the set $X$.

If all agents have the same preferences, then $P O(P)$ must contain all allocations. So, this means there is at least one good belonging to $X\left\{x_{\gamma}\right\}$ for which agent $\delta$ and other agents must have different preferences. 